\title{
ARM Based Design of Intrabody Communication System
}

\author{
${ }^{1}$ Sumit Kumar, ${ }^{2}$ Prof. M. U. Inamdar \\ ${ }^{I}$ Department Of Extc, Scoe, Sudumbare, Pune, India \\ ${ }^{2}$ Department Of Extc, Scoe, Sudumbare, Pune, India
}

\begin{abstract}
Intrabody communication (IBC) is a new data transmission concept that uses human body as a communication channel to transmit data. One of the main objectives of research into intrabody communication is the characterization of the human body as a transmission medium for electrical signals. And these characterization is strongly influenced by the conditions under which the experiments are performed. This paper explains a different methodology for IBC from the existing one. In this paper we have explained a method of data transmission between two personal computers through human body using serial data communication protocol. Further we have investigated its feasibility for distant range, about transceiver design and its future uses.
\end{abstract}

Index Terms: Intrabody communication (IBC), serial communication, Human Area Network (HAN), GUI.

\section{Introduction}

Intrabody communication is a technique that uses human body as a channel to transmit electrical signals. IBC is based on near field coupling mechanism in which majority of the signal is confined through the surface of body thereby least energy being radiated into the air. While in the case of different RF standards such as Bluetooth and Zigbee electromagnetic energy get radiated in the environment causes more power consumption. IBC application area is very wide. It can be used for communication between mobile terminals as well as for communication between two mobile terminals, between a mobile terminal and a terminal embedded in the environments and between the other terminals embedded in the environments.

The typical advantages of intrabody communication are as follows: first, cables are eliminated. Second, communication can easily be started or terminated at will as communication channels are formed only when a person touches terminals. Third, it is more secure than ordinary wireless communication because data signals are not radiated outward as it is confined to the body.

These advantages have led researchers to improve IBC electronic prototypes [1]-[3] as well as to define new applications [4], [5]. Therefore, IBC is a promising approach capable of covering some of the main technical challenges that are yet to be resolved in BSN [6], such as the requirement of small-size, power-saving, and miniaturized intelligent wearable devices.

A variety of IBC techniques have been proposed, which can be grouped into three main approaches: galvanic coupling [7], [8], capacitive coupling [9], [10], and waveguide methods [11]. The first two rely on the coupling of low frequency, low-level currents and voltages into the human body, respectively, whereas in the last technique, an electromagnetic wave propagates through the body, which is commonly associated with the use of higher frequencies, thus involving a non negligible radiation component into the air.

\section{Related Works}

The concept of IBC was originally proposed by Zimmerman [12]. He demonstrated how mobile devices near the human body can exchange digital information by capacitive coupling in Pico ampere currents through the human body. Zimmerman said that, the near-field communication can operate at very low frequencies and low transmission power. The prototype of the PAN transmitter operates at $330 \mathrm{kHz}, 30 \mathrm{~V}$, with a transmission power consumption of $1.5 \mathrm{~mW}$ for charging the electrode capacitance. Direct coupling by Masaaki Fukumoto [13] et al is a modified version of the basic capacitive method. The system operates by analog frequency modulation at frequencies within $50 \mathrm{kHz}$ to $90 \mathrm{kHz}$ for transmitting a simple protocol of ID numbers. Sasaki [14] et al tried to illuminate the principles of intrabody communication, where the Electro Optic [EO] sensor is used to receive data signal. Maria Amparo Callej'on [15] et al implemented galvanic [16], [17] and capacitive coupling [18], [19] setups and carried out comprehensive set of measurements by analyzing fundamental IBC parameters such as optimum frequency range, maximum channel length and type of electrodes. MirHojjat Seyedi [20] et al done a survey to examine the ongoing research in the area of Intrabody communication for body area network applications and highlights IBC fundamentals, current mathematical models of the human body, IBC transceiver designs. It is found that IBC is a new short range non-RF wireless communication technique specified by the IEEE 802.15.6 using the human body as a transmission medium. IBC 
technique potentially provides a more power efficient and naturally secure short range communication method for body area networks, compared to wireless RF.

\section{Proposed Method}

This section describes the proposed model of Intrabody communication in detail. In this model we have considered communication between two personal computers through human body.

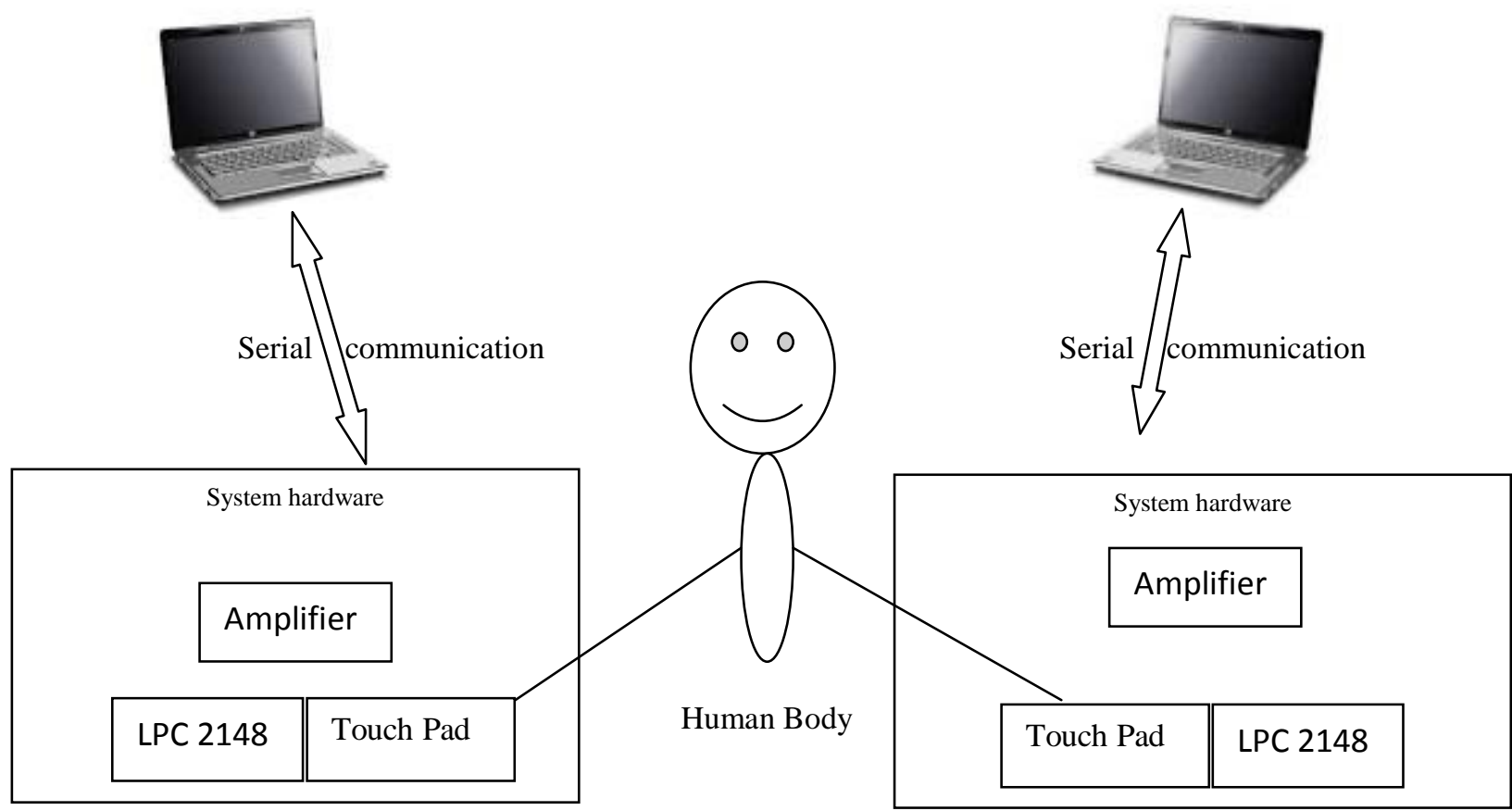

Fig. 1. Intra Body Communication System model

\section{A. System Hardware Model}

On the hardware side of system we have used windows based personal computer (PC). On PC a graphical user interface (GUI) is created. By using serial data transmission protocol between PC and the hardware data can be transmitted as shown in Fig.1. ARM 7 LPC2148 is at the core of the system. It works at $3.3 \mathrm{~V}$ power supply and for that a LM 1117 is used. To increase the range of communication LM 358 can be used.

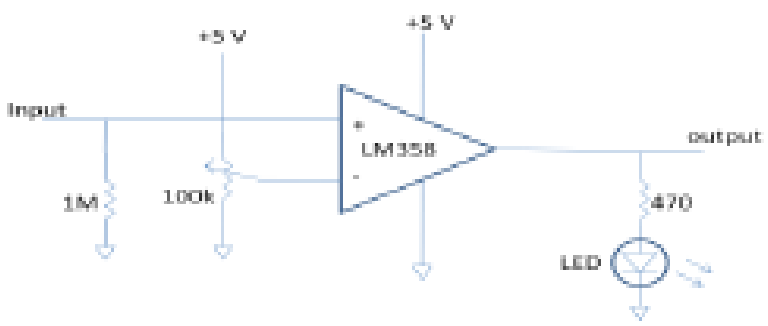

Fig.2. LM 358 Amplifier

Input to the amplifier is current (in $\mathrm{mA}$ ) that comes from body. With the help of $1 \mathrm{M}$ resistor current to voltage conversion takes place and by using 100k potentiometer reference voltage can be adjusted. In the fig. 1 the system hardware shown is basically a transceiver. It can transmit and receive data.

\section{B. System Software model}

System software is divided into two parts; one is on the processor side and other is on the PC side. For the processor side we have used the embedded $\mathrm{C}$ in the keil software and for creating GUI on PC we have used the VB.net. 
For the processor, program is written in embedded C. Data transmission is defined through USB TTL from PC through processor to touchpad. The personal data will be received from UART 1 and passed to the touchpad through UART 0. The data flow diagram on processor side is shown in fig.3.

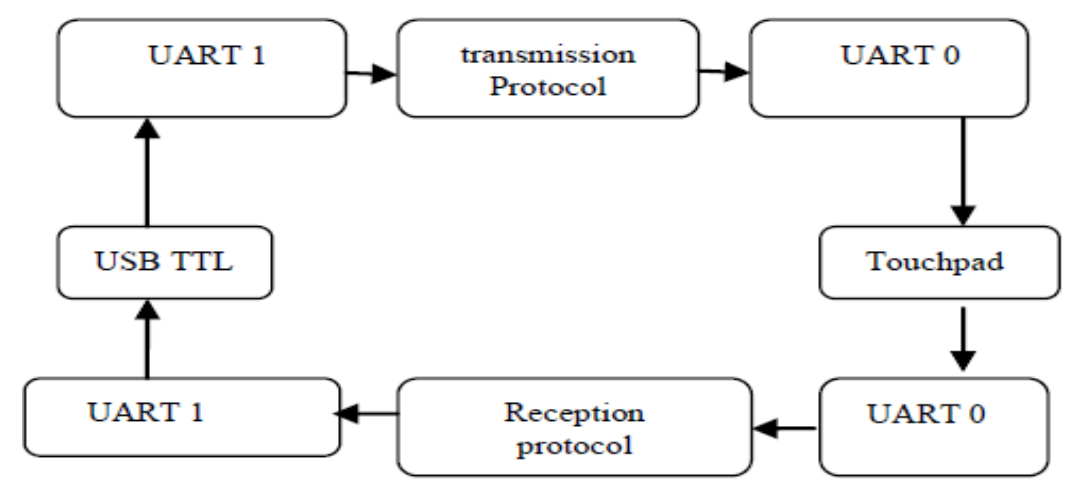

Fig.3 Data flow diagram for Processor

On the PC side, GUI elements such as text field and different buttons for data transmissions are created and are filled with all required data. Establishment of connection between PC device and system hardware is done by selecting Tx or Rx buttons which are created on GUI.

The data flow diagram shown in fig.4 itself explains the flow of information from PC to external hardware.

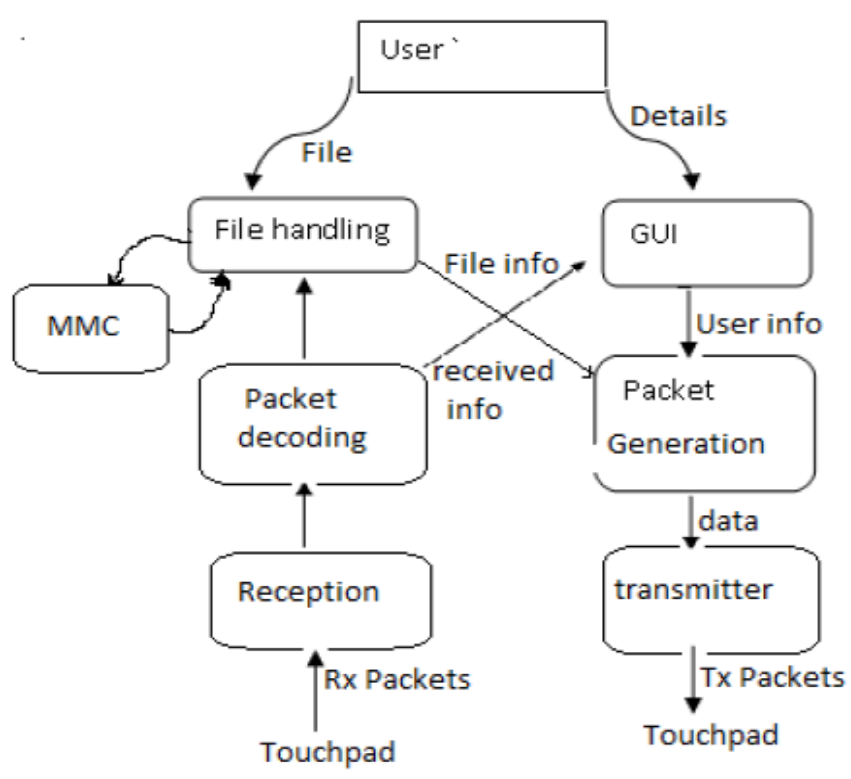

Fig.4 Dataflow diagram to explain data transmission between PC and system hardware

According to data flow diagram as shown in fig. 4, a user fills the information in the GUI form or read the file from the MMC through the file handling system. Then the data or file is going to be converted in the packets. Those packets are transmitted to the receiver controller through the body. At the time of reception the receiver controller receives the data from the body. The received data is decoded and if the data is information then it will send to the GUI and it will display on it. If the data is file then it is given to the file handing system and stored into the MMC. 


\section{Result And Discussion}

So with the help of designed GUI and its elements as shown in fig.5, it is possible to transmit personal data and file from SD card. First system serial data path availability will be checked, once it is confirmed connection will be established between PC and the system hardware through serial port of PC.

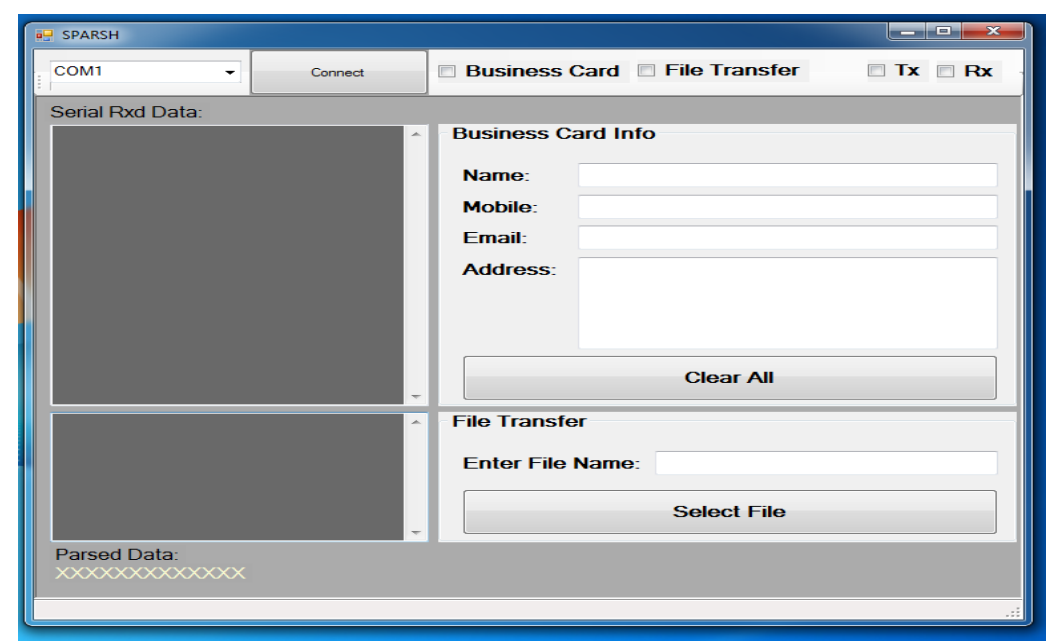

Fig. 5. Designed GUI on PC

In this paper we have developed methodology for the transmission of data of 255 byte. The range between two mobile devices can be improved with the help of amplifier. We have used a standard serial data communication rate of 9600 baud rate.

\section{Conclusion And Future Scope}

IBC is a new short range non-RF wireless communication technology specified by the IEEE 802.15.6 using human body as a transmission medium. Many authors have presented the issues and challenges of this field in their papers. Still research is going on to address different issues. In our paper we have tried to develop an external transceiver hardware for transmission of data between two computers.

Further in future we can place designed external hardware inside the system. Data transmission security can be provided . Video, audio or image file can also be transmitted with increased data rate.

Further the rapid increase in healthcare demand has also seen novel developments in health monitoring technologies such as body area network (BAN) paradigm. BAN technology consists of a network of sensors which operates continuously and measure critical physical and physiological parameters like mobility, heart rate and glucose level. So the Intrabody communication is an alternative option in BAN.

\section{References}

[1] L. Wang, G.-Z. Yang, J. Huang, J. Zhang, L. Yu, Z. Nie, and D. R. S. Cumming, "A wireless biomedical signal interface systemon-chip for body sensor networks," IEEE Trans. Biomed.Circuits Syst., vol. 4, , pp. 112-117, Apr. 2010.

[2] H.-Y. Shih and Y.-C. Chang, "68.4 $\mu \mathrm{W} 400 \mathrm{MHz}$ intrabody communication receiver front-end for biomedical applications," Electron Lett, vol. 48, no. 3, pp. 143-144, Feb 2012

[3] Y. Song, K. Zhang, B. Kang, R. Xie, F. Shang, and Q. Hao, "the sensitivity characteristics of the electrostatic coupling intra-body communication based on the Mach-Zender electro-optic modulation,"in communications in computer and Information Science, vol. 202. Berlin, Germany: Springer, 2011, pp. 210-216

[4] S. Pun, Y. Gao, P. Mak, H. Ho, K. Che, H. Ieong, H. Wu, M. Vai, and M. Du, "Galvanic intrabody communication for affective acquiring and computing,” IEEE Trans. Affective Comput., vol. 3, no. 2, pp. 145-151, Apr.- Jun. 2012.

[5] M. Fujikawa and M. Nishigaki, "A study of prevention for social engineering attacks using real/fake organization's uniforms: Application of radio and intra-body communication technologies," in Proc. 6th Int Conf. Availability, Reliab. Security, 2011, pp. 597-602.

[6] L. Shasha, H. Fengye, and L. Guofeng, "Advances and challenges in body area network," in Communications in Computer and Information Science, vol. 226. Berlin, Germany: Springer, 2011, pp. 58-65.

[7] M. S. Wegmueller, A. Kuhn, J. Froehlich, M. Oberle, N. Felber, N Kuster, and W. Fichtner, "An attempt to model the human body as a communication channel,” IEEE Trans. Biomed. Eng., vol. 54, no. 10, pp. 1851-1857, Oct. 2007.

[8] Y. Song, Q. Hao, K. Zhang, M. Wang, Y. Chu, and B. Kang, "The simulation method of the galvanic coupling intrabody communication with different signal transmission paths," IEEE Trans. Instrum. Meas., vol. 60, no. 4, pp. 1257-1266, Apr. 2011.

[9] R. Xu, H. Zhu, and J. Yuan, "Electric-field intrabody communication channel modeling with finite element method," IEEE Trans. Biomed Eng., vol. 58, no. 3, pp. 705-712, Mar. 2011.

[10] Z. Lucev, I. Krois, and M. Cifrek, "A capacitive intrabody communication channel from $100 \mathrm{kHz}$ to $100 \mathrm{MHz}$," in Proc. IEEE Instrum. Meas. Technol. Conf., May 2011, pp. 1-4. 
[11] J. Wang, Y. Nishikawa, and T. Shibata, "Analysis of on-body transmission mechanism and characteristic based on an electromagnetic field approach,” IEEE Trans. Microw. Theory Tech., vol. 57, no. 10, pp. 2464-2470, Oct. 2009

[12] T. G. Zimmerman, "Personal area network (PAN)," M.S. thesis, Media Lab., Massachusetts Inst. Technol., Cambridge, Mar. 1995

[13] Mitsuru Shinagawa, Masaaki Fukumoto, Katsuyuki Ochiai, and Hakaru Kyuragi, "A Near-Field-Sensing Transceiver for Intrabody Communication Based on the Electro optic Effect," IEEE Transactions On Instrumentation And Measurement, vol. 53, no. 6, pp. 1533-1538, Dec. 2004

[14] Ai-ichiro Sasaki, Mitsuru Shinagawa, Katsuyuki Ochiani, "Principles and Demostrastion of intrabody communication with a sensitive electro optic sensor," IEEE Transactions on Instrumentation and Measurement, vol. 58, no. 2, pp. 457-466, Feb. 2009

[15] Maria Amparo Callej’on, David Naranjo-Hern'andez, Javier Reina-Tosina, and Laura M. Roa, "A Comprehensive Study into Intrabody Communication Measurements," IEEE Transactions On Instrumentation And Measurement, vol. 62, no. 9, pp. $2446-2455$ Sep. 2013.

[16] M. S. Wegmueller, A. Kuhn, J. Froehlich, M. Oberle, N. Felber, N. Kuster, and W. Fichtner, "An attemp to model the human body as a communication channel,” IEEE Transactions On Instrumentation And Measurement, vol. 54, no. 10, pp. 1851-1857, Oct. 2007.

[17] Y. Song, Q. Hao, K. Zhang, M. Wang, Y. Chu, and B. Kang, " The simulation method of the galvanic intrabody communication with different signal transmission path," IEEE Transactions On Instrumentation And Measurement, vol. 60, no. 4, pp. 1257-1266, Apr. 2011.

[18] R. Xu, H. Zhu, and J. Yuan, "Electric-field intrabody communication channel modelling with finite element method," IEEE Transactions on Instrumentation and Measurement, vol. 58, no. 3, pp. 705-712, Mar. 2011.

[19] Z. Lucev, I. Krois, and M. Cifrek, "A capacitive intrabody communication channel from $100 \mathrm{kHz}$ to $100 \mathrm{MHz}$," in Proc. IEEE Instrum. Meas. Technol. Conf., May 2011, pp. 1-4.

[20] MirHoijat Seyedi,Behailu Kibret, Daniel T. H. Lai, and Michael Faulkner, Member, IEEE, "A Survey on Intrabody Communications for Body Area Network Applications," IEEE Transactions On Biomedical Engineering, vol. 60, no. 8, pp. 20672079, Aug. 2013

[21] Brian W. Kernighan, Dennis M. Ritchie, The C programming Language, First Edition 1988, Prentice-Hall, ISBN 0-13-110370-9. http://www.cadsoftusa.coom/download-eagle/

[22] Ramakant A. Gayakwad, Op-Amps and Linear Integrated Circuits, 4thEdition, Prentice-Hall.

[23] Robert L. Boylestad, Louis Nashelsky, Electronic Devices and Circuit Theory, 10thEdition, Prentice-Hall. 